\title{
Input, Output and Construction Methods for Custom Fabrication of Room-Scale Deployable Pneumatic Structures
}

\author{
SAIGANESH SWAMINATHAN, Carnegie Mellon University, USA \\ MICHAEL RIVERA, Carnegie Mellon University, USA \\ RUNCHANG KANG, Carnegie Mellon University, USA \\ ZHENG LUO, Carnegie Mellon University, USA \\ KADRI BUGRA OZUTEMIZ, Carnegie Mellon University, USA \\ SCOTT E. HUDSON, Carnegie Mellon University, USA
}

\begin{abstract}
In this paper, we examine the future of designing room-scale deployable pneumatic structures that can be fabricated with interactive capabilities and thus be responsive to human input and environments. While there have been recent advances in fabrication methods for creating large-scale structures, they have mainly focused around creating passive structures. Hence in this work, we collectively tackle three main challenges that need to be solved for designing room-scale interactive deployable structures namely - the input, output (actuation) and construction methods. First, we explore three types of sensing methods - acoustic, capacitive and pressure - in order to embed input into these structures. These sensing methods enable users to perform gestures such as knock, squeeze and swipe with specific parts of our fabricated structure such as doors, windows, etc. and make them interactive. Second, we explore three types of actuation mechanisms - inflatable tendon drive, twisted tendon drive and roll bending actuator - that are implemented at structural scale and can be embedded into our structures to enable a variety of responsive actuation. Finally, we provide a construction method to custom fabricate and assemble inter-connected pneumatic trusses with embedded sensing and actuation capability to prototype interactions with room-scale deployable structures. To further illustrate the collective (input, output and construction) usage of the system, we fabricated three exemplar interactive deployable structures - a responsive canopy, an interactive geodesic dome and a portable table (Figures 1 and 2). These can be deployed from a compact deflated state to a much larger inflated state which takes on a desired form while offering interactivity.
\end{abstract}

CCS Concepts: • Human-centered computing $\rightarrow$ Human computer interaction (HCI);

Additional Key Words and Phrases: deployable pneumatic structures; digital fabrication; embedded interactivity

ACM Reference Format:

Saiganesh Swaminathan, Michael Rivera, Runchang Kang, Zheng Luo, Kadri Bugra Ozutemiz, and Scott E. Hudson. 2019. Input, Output and Construction Methods for Custom Fabrication of Room-Scale Deployable Pneumatic Structures. Proc. ACM Interact. Mob. Wearable Ubiquitous Technol. 3, 2, Article 62 (June 2019), 17 pages. https://doi.org/10.1145/3328933

\section{INTRODUCTION}

Recent work has explored a range of new fabrication technologies to rapidly create custom functional interactive objects with printed electronics [19], optics [28], metamaterials [9], etc. Over time, the space of materials that can be fabricated and types of interactive sensing, display, and actuation that can be created have been expanded

Contact Authors' addresses: saiganes@cmu.edu, Human-Computer Interaction Institute, 5000 Forbes Avenue, Pittsburgh, PA-15213, USA.

Permission to make digital or hard copies of all or part of this work for personal or classroom use is granted without fee provided that copies are not made or distributed for profit or commercial advantage and that copies bear this notice and the full citation on the first page. Copyrights for components of this work owned by others than ACM must be honored. Abstracting with credit is permitted. To copy otherwise, or republish, to post on servers or to redistribute to lists, requires prior specific permission and/or a fee. Request permissions from permissions@acm.org.

(c) 2019 Association for Computing Machinery.

2474-9567/2019/6-ART62 \$15.00

https://doi.org/10.1145/3328933

Proc. ACM Interact. Mob. Wearable Ubiquitous Technol., Vol. 3, No. 2, Article 62. Publication date: June 2019. 

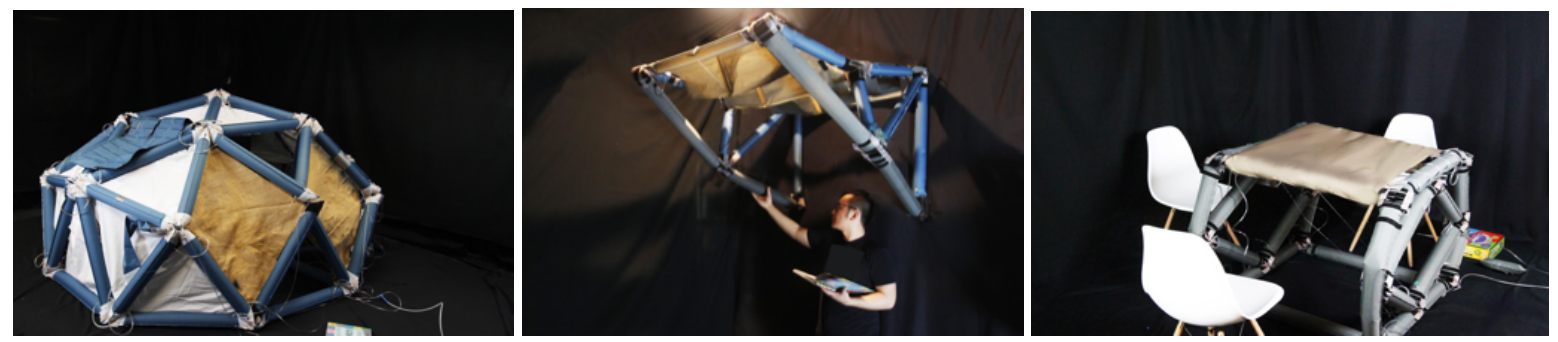

Fig. 1. (a) A geodesic dome that deploys itself upon inflation and has embedded actuation and acoustic sensing to open its doors and raise its windows; (b) a canopy with a controllable venetian blind via capacitive sensing; (c) a portable table inflated on-demand via a pressure sensitive control. All of our structures have embedded actuation and sensing that support a range of interactions (e.g., a user performs a knock gesture on the dome's door and the door swings open).

to include a wide range of functional interactive objects. This has increased our ability to create truly customized solutions to user problems, needs, and desires, as well as more generally democratized the production of interactive physical artifacts. However, much of this work has concentrated on the production of interactive devices at what might be called a "hand" scale. Much less attention has been paid to creating interactive objects at a larger scale: what we might call "room" scale (or larger). In the work presented here, we seek to explore interaction with a class of objects that can be custom fabricated at this scale: pneumatically inflated deployable structures. Our addition of interactivity allows these structures to move from supporting environments constructed with passive forms, to interactive forms which can respond to user inputs, and thus create responsive environments.

\section{MOTIVATION AND CONTRIBUTION}

Most structures around us are built and then, often with considerable effort, retrofitted with custom sensors, IoT devices, and actuation (e.g., automated doors/windows with proximity sensors). Our overarching goal is to develop techniques for fabrication of sensors and actuators as an integral part of the structure manufacturing processes itself rather than added later.

We do this in the context of deployable structures [5] which offer further responsiveness and flexibility in an environment since they can be deployed and removed on demand. In general, deployable structures include those which have a compact (undeployed) state that can be expanded into a larger and/or more functional configuration. Many scenarios exist with interactive deployable structures where inferring user context and providing support for interaction can improve user experience. A non-exhaustive list of example scenarios includes a storage box structure that opens and retracts when a correct key-code is pressed; blinds which sense weather conditions and automatically open and close; a self-deploying table which expands when it senses multiple users; an interactive chair with controls to adjust backrest, etc.

In this work, we have chosen to consider inflatable structures of this type for a number of reasons. Inflatable structures often offer a large expansion ratio from their compact to deployed states. Many useful objects can be custom fabricated using comparatively easy to work with materials, and manageably small fabrication machines; and the deployment of these structures is fully reversible so that they can be deployed and redeployed on demand. To explore the interactive possibilities around this space of objects, we introduce several techniques for basic input sensing, as well as actuation mechanisms and a holistic construction method for collectively (sensors, actuators and structures) putting together room-scale interactive deployable structures, i.e., structures which can respond to human input and implement interactions. We also consider several example objects which can be built using these techniques (Figure 1). 

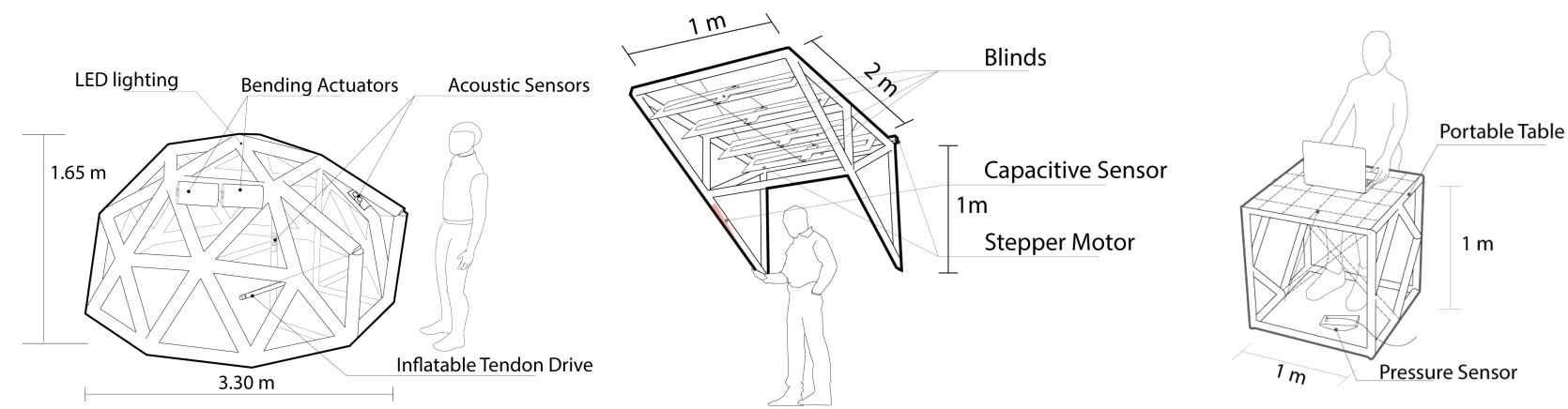

Fig. 2. (a) An overview of an interactive geodesic dome with various actuators and sensors embedded as a part of the structure; showing the placement of contact microphones that are used to detect user gestures via swept frequency ultrasonic acoustic sensing; the various actuator mechanisms for door and windows (deployed) (b) Overview of interactive canopy structure with an embedded capacitive sensor for controlling blinds. (c) Portable table with embedded pressure sensing for deployment.

To this end, we contribute a toolbox of basic sensing and actuation methods, that designers can use with our uniform and relatively simple structural manufacturing process. Main requirements for input sensing were employing materials already in use and supplying a reasonably rich set of basic primitive, reusable, interaction techniques. Hence we selected a wide variety of sensing methods - capacitive sensing [7], swept frequency ultrasonic sensing [13] and pressure sensing [8] - that can be adapted to work with our structural members (trusses).

Similarly, we contribute three classes of soft actuators for manipulation of structures: linear (increase/decrease in length) used in pull/push of articulated joints, bending and twisting. Our actuators are inspired by a growing body of soft robotic literature such as pouch motors [18], gait robots [26] and continuum arm robots [24] where the actuators have been thoroughly modeled and tested with materials such as PDMS (commonly referred to as silicon rubber). Note that, we extend this literature by implementing them at a structural scale using our processes and provide ways for designers to utilize them as actuators in interactive structures. In addition, we consider two technical evaluations, one measuring the accuracy of our swept frequency acoustic sensing gesture input approach (which relies heavily on a machine-learned recognizer), and a second evaluation which considers aspects of structural strength for these types of structures. Finally, we perform an informal gesture elicitation study and sketching session with subjects who are expert designers to explore the design space of interaction techniques opened by our basic input sensing methods.

Although we have constructed, and will briefly describe, a custom fabrication machine for our inflated structures, it should be noted that the focus of the work presented here is centered on engineering the collective capabilities emerging from sensing, actuation and construction methods, rather than the advances of the fabrication methods or any in-depth improvements of each individual technique.

We believe that collectively our sensing, actuation, and structure manufacturing methods provide a resulting system that demonstrates new and interesting HCI capabilities and present a viable path for manufacturing deployable smart structures with built-in interaction at room-scale. To illustrate this, we present three example objects that embed our proposed sensing and actuation capabilities - an interactive geodesic dome (described below), a responsive canopy and a deployable portable table (Figures 1 and 2). 


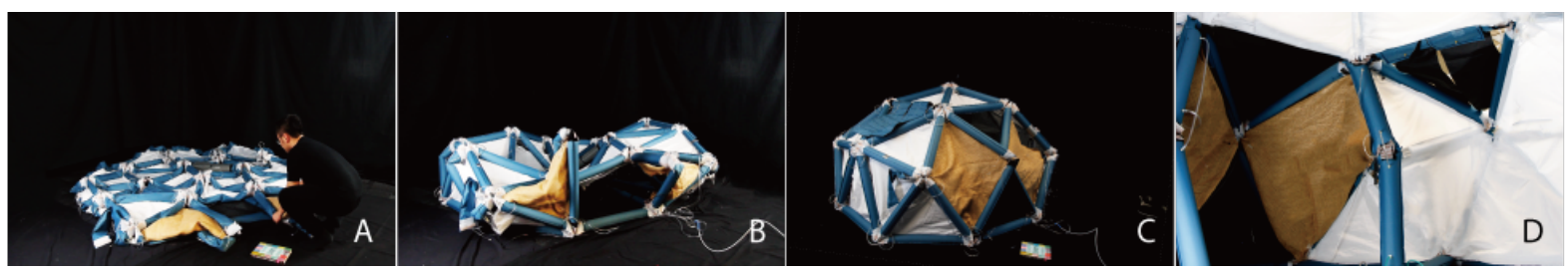

Fig. 3. Shows a user connecting a pneumatic pipe (a) and the structure inflating itself (b) the structure is fully deployed (c) and the structure from inside $(\mathrm{d})$.

\subsection{Example: Interactive Geodesic Dome}

As a motivating example, we now describe how our interactive geodesic dome structure works and various interactions with the structure. Additional examples are further described in detail in Section 6

Our geodesic dome consists of actuated doors and windows as well as acoustic sensors that can pick up user gestures as a part of the structure (Figure 2a). Specifically, we perform swept frequency ultrasonic acoustic sensing by embedding contact microphones on the door and a support beam inside the structure. We are able to perform acoustic sensing for any truss member from a single point. We fabricate all our structures with inter-connected pneumatic truss members, allowing users to deploy large scale structure from a flat configuration by simply connecting an air supply (Figure 3).

After inflating the geodesic dome, the user performs a knock gesture on the door (Figure 4a). Upon the knocking, our sensing/machine learning pipeline recognizes the user's gesture and actuates the door, allowing the user to enter (Figure $4 \mathrm{~b}$ ).

After entering the door, the user squeezes (Figure 4c) an inflated member and our system is able to recognize the gesture and actuate windows by rolling them up (Figure 4d).

Similarly, if the outside environment gets dark, a user can perform the swipe gesture (Figure 4e), our system will respond by turning the lighting on inside the dome (Figure $4 \mathrm{f}$ ).

\section{RELATED WORK}

Our work builds on many interesting emerging areas in $\mathrm{HCI}$ such as fabricating human-scale structures, sensing with soft materials and interacting with large scale architecture.

\subsection{Fabricating Passive Room-Scale Structures}

A growing number of researchers in $\mathrm{HCI}$ community and other relevant fields have started investigating how to scale up fabrication processes for larger structures. One body of work looks to guide human workers to assemble structures with hand-held tools and feedback. For example, Yoshida et al. [32], guided human workers with projection mapping and hand-held dispensers, that used chopsticks and glue as the basic construction material. Similarly, in the crowdsourced fabrication project [12] human workers were guided to different locations of a structure with a LED device embedded in the structure itself.

A second approach is to use robotic arms and mobile robots to scale-up fabrication. For example., Gramazio and Koehler [6] fabricated building facades by extruding filament in 3D space using robotic arms. Similarly, Mataerial [1] is a project where freeform fabrication was achieved using a robotic arm to cure polymers while printing. Minibuilders [2] uses mobile robots for printing along a driven path and building up the foundation footprint of buildings along that route.

Finally, other researchers have studied how to enable users to come up with their own designs of structures, print and assemble them using desktop 3D printers. For example, Luo et al. [15] designed Chopper an interactive 

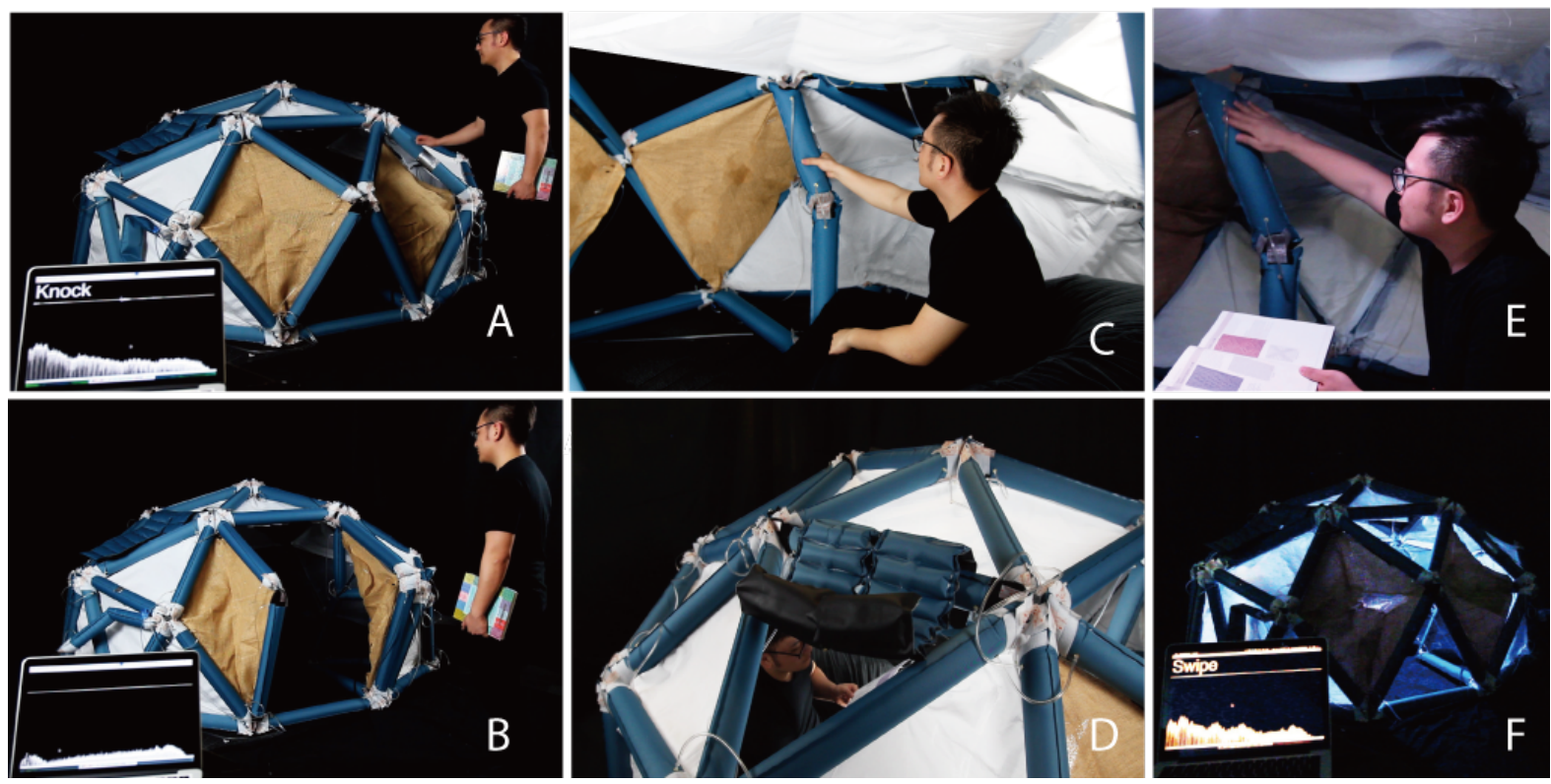

Fig. 4. (a) shows a user performing a knock gesture and our system recognizing user input; (b) shows the door opening for the user to enter.(c) shows a user performing a squeeze gesture; (d) shows the windows of the dome rolling up in response.(e) shows a user performing a swipe gesture; (f) shows the lighting switched on inside the dome.

system that lets users decompose a large 3D object into smaller parts so that each part fits into the printing volume of a desktop 3D printer. Trussfab [11] is a system that enables users to create own versions of trusses, print joint connectors and assemble them using plastic bottles. More recently, and forming the important motivation for the work here, Printflatables [25] introduced machinery and design primitives for fabricating human-scale pneumatic objects as a single component. In contrast, our approach combines truss designs with pneumatics to fabricate room-scale deployable structures, i.e., we use pneumatic tubes that are pre-connected to form truss members, but with an emphasis on making these structures interactive and responsive rather than passive.

\subsection{Sensing in Flexible Objects}

A range of techniques exists that explore sensing on soft surfaces. The most relevant prior efforts are situated in sensing of flexible, shape-changing interfaces. PneUI [30] demonstrates how sensing materials like liquid metal and conductive foil can be embedded into soft pneumatically actuated composites to create sensors in PDMS (commonly referred to as Silicone Rubber) at hand scale. Similarly, Aeromorph [20] examines how changes in air pressure can be sensed in heat-sealed inflatable objects. While our work features sensing of heat-sealed inflatable objects, we focus on fabricating larger structures and demonstrate a wider range of sensing capabilities beyond air pressure changes.

Other work in the HCI community has fabricated flexible sensors using conductive inks embedded into deformable 3D printed objects [3] or absorbed into soft materials like sponges [17]. Another approach examines impregnating elastomers with carbon particles to create piezoresistive sensors [31]. In the domain of textiles, researchers have explored embedding conductive textiles into other soft materials (e.g. fabrics) to create sensing opportunities on and around the body [14, 23, 27]. Our work extends these approaches by demonstrating how 

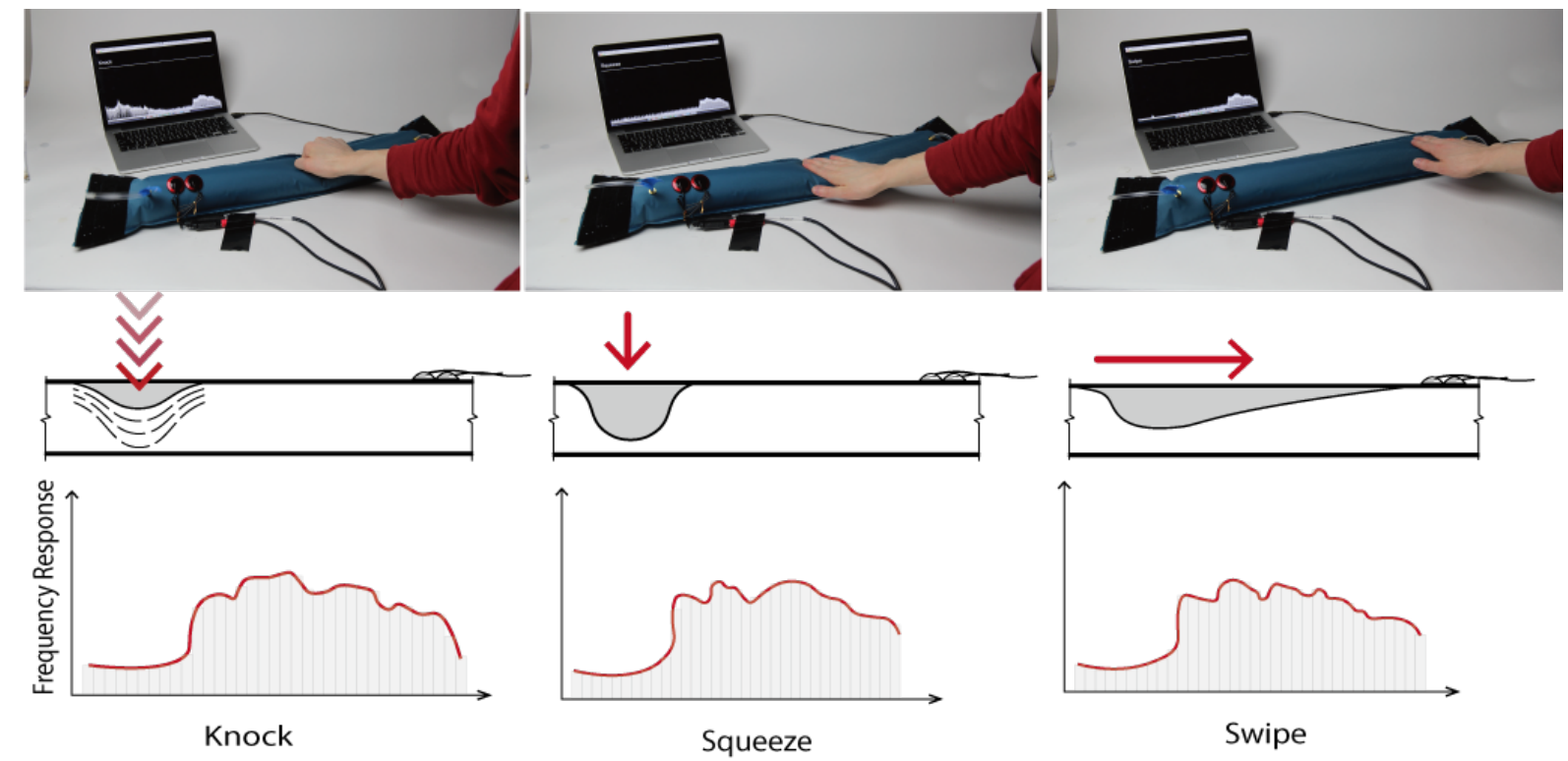

Fig. 5. Using swept frequency ultrasonic acoustic sensing, we are able distinctly identify a number of gestures when performed on our inflated truss members. Note that the frequency responses above refer to the ultrasonic region (displayed in the far-right of the laptop screen).

similar sensing capabilities, including swept frequency ultrasonic acoustic sensing [13], can be integrated into deployable, room-scale pneumatic structures.

\subsection{Interaction with Infrastructure}

Researchers have also examined how to scale interaction to larger scale surfaces and infrastructure. For example, GravitySpace [4] looked at how to estimate and sense different poses of users on the floor using FTIR and a camera located below the floor. Paradiso et al. [21] used a scanned laser rangefinder operating parallel to a wall's surface to detect hand touches. BaseLase [16] provided an interactive context+focus laser projected floor that users can interact with using cameras to track users' positions and poses.

Other sensing approaches related to our methods involve using a contact microphone with room-scale objects such as windows [22], ping-pong table [10] to perform acoustic time of arrival sensing. More recently, Wall++ [33] looked at how to allow walls to become smart infrastructure and perform context aware sensing by electric field tomography near a wall augmented with a painted on antenna array, as well as sensing of electromagnetic noise in a space.

\section{INPUT METHODS}

In this section, we describe the various input mechanisms that can be embedded in inflated deployable objects in our system to support interaction. Our structures rely on a combination of input from acoustic, capacitive and pressure sensing. Our example applications integrate these input mechanisms into the structure for users. 


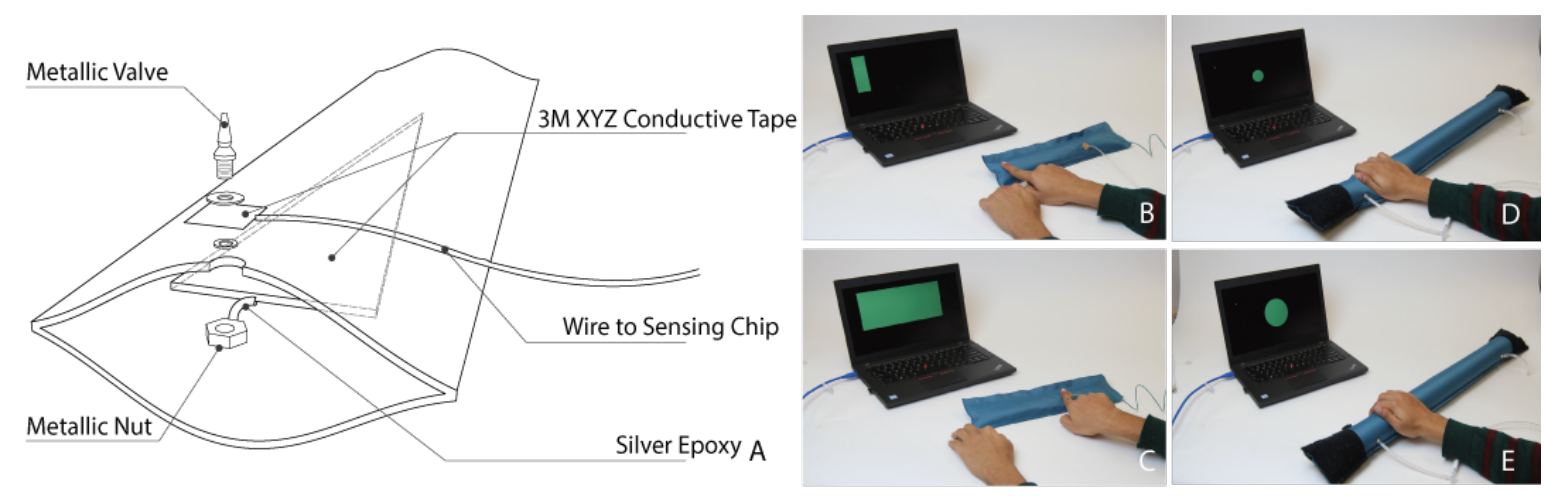

Fig. 6. (a) Technical drawing of our capacitive slider implementation embedded into the inflatable member; (b) shows a user beginning their finger along member; (c) shows the visualized output on a laptop display (d \& e) Our pressure sensing implementation: as a user applies force to the inflated member and the pressure is visualized on the laptop display.

\subsection{Requirements for Sensing}

The main requirements for integrating sensing with inflatable structures were (1) employing materials already in use, or readily adaptable to the production in this form, and (2) supplying a reasonably rich set of primitive, reusable, interaction techniques. We propose three types of sensing in this work: ultrasonic acoustic, capacitive and pressure sensing. The application of these methods (either individually or together) should be based on the context of use. For instance, pressure sensing needs regulated air pressure to work well, whereas capacitive sensing can work well even in low pressure conditions. Similarly, in a noisy environment (such as sidewalks or roads with traffic) capacitive sensing may be favorable to using swept frequency ultrasonic acoustic sensing. However for situations/applications where inflatable objects are used in water (such as in a pool), capacitive sensing might not work. For more details on various application scenarios, please check section 8.2 for results from the design workshop. In the next section, we go over the engineering details of each sensing implementation.

\subsection{Ultrasonic Acoustic Sensing}

We implemented swept frequency ultrasonic acoustic sensing using an approach similar to SweepSense [13]. We use two small contact piezo elements that are affixed to the back of an inflated truss. One serves as a speaker and repeatedly emits a near ultrasonic frequency sweep (18 to $24 \mathrm{kHz}$ within a 100 millisecond window) while the other acts as a microphone and receives the resulting attenuated signal. Depending on how the truss is manipulated, the internal configuration of the truss's cavity alters the attenuation of the signal at different frequencies as seen in (Figure 5).

We experimented with a number of gestures and found the most distinct responses came from a knock, squeeze, and swipe (Figure 5). From the responses, we extract a series of features: RMS, average power, the spectral center of mass, $\max / \mathrm{min}$ index and values, standard deviation, and spectral band ratios. We then train a Sequential Minimal Optimization-based Support Vector Machine (SMO-SVM) with default parameters using the Weka Toolkit. Our model is trained by capturing the responses on a single set of 10 trials for each of the 3 gestures. For more details on the classification accuracy please see the evaluation section below. 

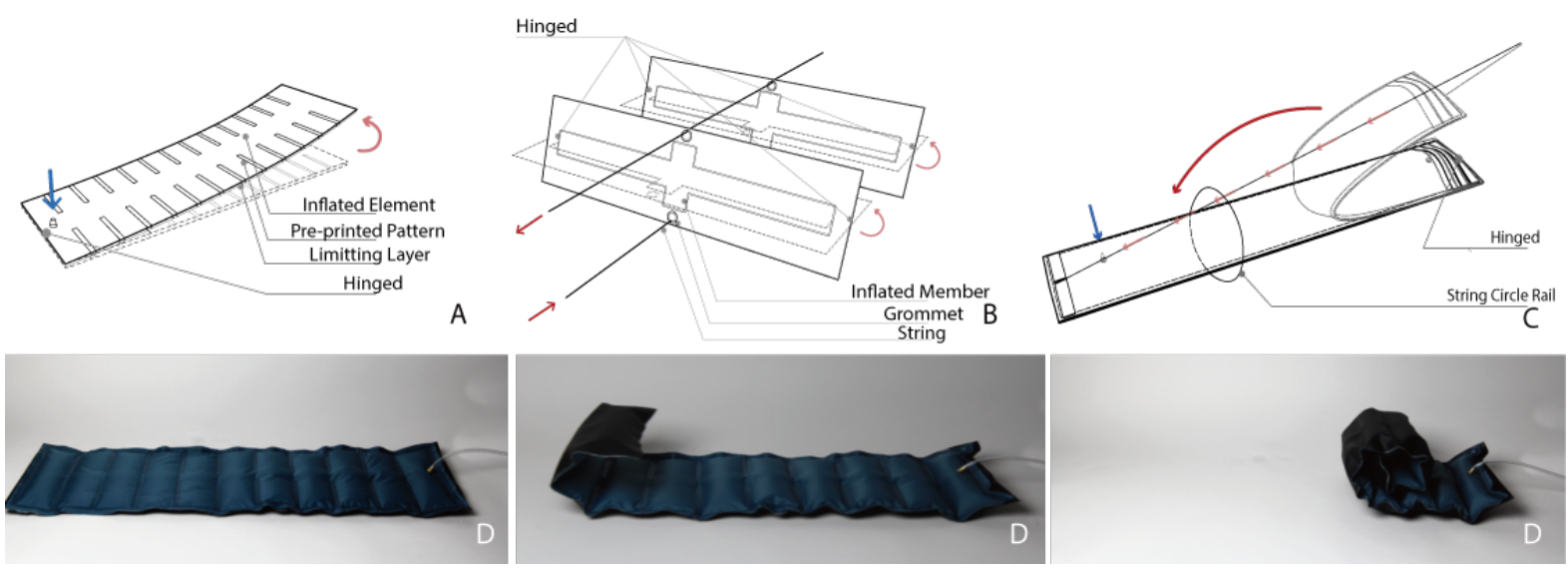

Fig. 7. (a) rolling bend actuator implementations (b) twisted tendon drive, and (c) Our inflated tendon drive. (d) Our rolling bend actuator shown in bottom half rolling upon inflation

\subsection{Capacitive Sensing}

We implemented a capacitive sensor in the form of a touch slider by embedding $3 \mathrm{M} \mathrm{XYZ} \mathrm{conductive} \mathrm{tape}{ }^{1}$ into our inflatable members during the fabrication process. As seen in (Figure 6a), we cut triangular patterns of $3 \mathrm{M}$ conductive tape and sealed it between our thermoplastic fabric layers. We further made connections between the tape and a metallic inlet valve by using silver epoxy. From the outside, we connected the valve to an MPR121 capacitive sensing board ${ }^{2}$ (which has 12 sensing pins sampling at a rate of $29 \mathrm{~Hz}$ ) controlled by an Arduino.

\subsection{Pressure Sensing}

We used an off-the-shelf pressure transducer (MPX57000-ASX ${ }^{3}$ ) for absolute pressure sensing. The sensor is able to detect the pressure changes in the range from $2.18 \mathrm{psi}$ to $101.53 \mathrm{psi}(15-700 \mathrm{kPa})$. We sense changes in pressure using an Arduino as seen in (Figure $6 \mathrm{~d} \& \mathrm{e}$ ).

\section{ACTUATION MECHANISMS}

In this section, we describe the various structural mechanisms that can be used to embed actuation. We experimented with three different output mechanisms that can actuate as part of the structure (Figure 7). The first two mechanisms (inflated tendon and twisted tendon) are inspired by continuum robotics [24] where articulated joints are actuated with strings.

\subsection{Inflated Tendon Drive}

Our inflated tendon drive consists of a truss member which can fold when deflated, thus releasing the tension on a tendon. Upon inflation, the truss straightens out and pulls on the tendon (Figure 7c). The fixed length of the tendon string makes the structure move by a predetermined amount. This mechanism is used in the geodesic dome example to open and close the door.

\footnotetext{
${ }_{1} 3 \mathrm{MXYZ}$ conductive tape: https://www.3m.com/3M/en_US/company-us/all-3m-products/ /3M-XYZ-Axis-Electrically-Conductive-Tape-9713/ ?N=5002385+3294001404\&rt=rud

${ }^{2}$ MPR121 capacitive sensing chip: https://www.sparkfun.com/datasheets/Components/MPR121.pdf

${ }^{3}$ Pressure Transducer: https://www.nxp.com/docs/en/data-sheet/MPX5700.pdf
} 

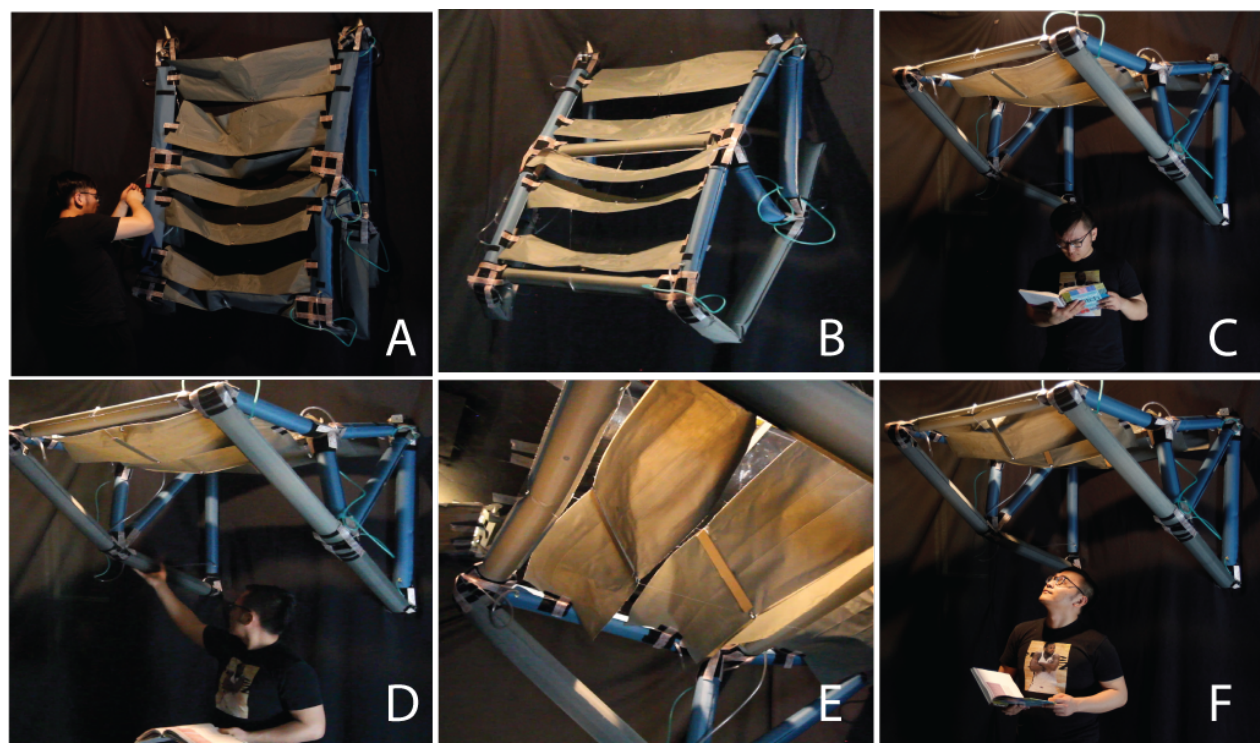

Fig. 8. A walk-through of our interactive canopy with a user deploying it ( $a-b)$ and controlling the blinds via a swipe gesture (c-d) to receive more light (e-f).

\subsection{Twisted Tendon Drive}

Twisted tendon drive is a combination of a tendon drive mechanism [24] with revolute joints. Typically, tendon drives are mechanisms that transmit motion through cables (only pull is possible through cables). In our specific implementation, the moment from the tendons is transferred to the joints which are located along the ends of the inflated members as seen in (Figure 7b). Since the joints are hinged, they act like revolute joints and allow the inflatable members to turn. This mechanism provides for variable actuation of the inflatable members perpendicular to their plane. To control the actuation, we simply move the tendon (or the string) attached.

\subsection{Rolling Bend Actuator}

We implemented a mechanism where inflation causes our fabrics to roll-up. We utilized the bending principle from Sheperd et al [26] to implement our mechanism. The printed mechanism consists of three layers of fabric, the first two layers are heat sealed in specific patterns (Figure 7a) to form inflatable pouches as seen in (Figure 7). The third layer is attached to the bottom of first two layers and acts as a strain limiting layer.

The direction of the bend will be away from the strain limiting layer, with each pouch contributing to the roll by pulling its neighboring pouches closer on the opposite side. The roll up can be seen in (Figure 7). Each pouch can bend to 75 degree angle (max), with standing pressure up to 16 PSI. The actuator was initially 33 inches long, shrinks to 23 inches.

\section{ADDITIONAL EXAMPLE STRUCTURES}

In this section, we show a few more additional demonstrator applications we developed by using the above input and actuation methods. First, we will walk through an interactive canopy with an embedded capacitive slider to control the blinds and a simple portable table that is deployed on pressure input. 

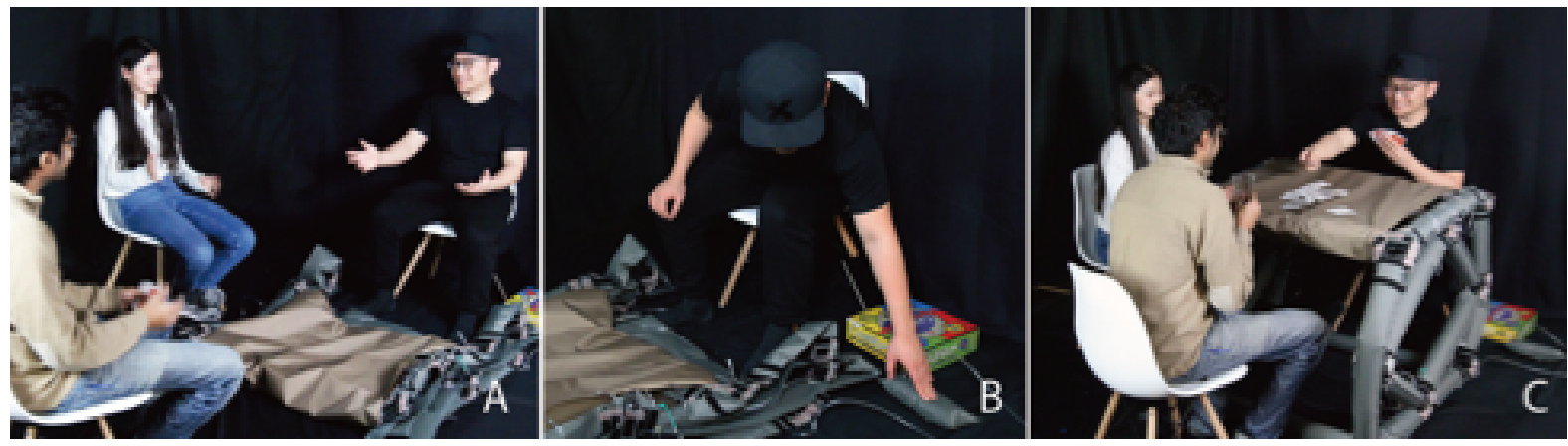

Fig. 9. A deployable table with a user triggering the structural members' inflation with an embedded pressure sensor.

\subsection{Deployable Responsive Canopy with Variable Blinds}

Our canopy consists of multiple truss frames connected to each other with inflated members. When deployed the canopy is $1 \mathrm{~m}$ tall, spans $1 \mathrm{~m}$ wide and stretches forward for $2 \mathrm{~m}$ (Figure $2 \mathrm{~b}$ ). The roof of the canopy consists of variable angle blinds, controlled by a twisted tendon drive actuation mechanism. The bottom truss member is embedded with a capacitive slider sensor to support user input (Figure 2b). To deploy the canopy, the user first hangs it as desired and makes a pneumatics connection (Figure 8a). Upon inflation, the canopy deploys itself (Figure 8b). Once fully deployed, the canopy provides a shade under which the user can stand and e.g., read a book (Figure 8c). The user then manipulates the embedded capacitive slider' (Figure 8d) on the truss member to control the amount of light. With the help of the twisted tendon drive mechanism, the blinds open (Figure 8e) e.g., to let more light in (Figure $8 \mathrm{f}$ ).

\subsection{Deployable Portable Table}

As a final demonstrator application, we implemented a deployable table with an embedded pressure sensor (Figure 9). First, we fabricated truss frames on both sides and connected them with beams to form our deployable table. When a user steps on the leg (truss member) of table, the truss members acts as a pressure sensor and the system simply senses the pressure change and triggers itself to deploy to inflation. The deployed table is $1 \mathrm{~m}$ tall and $1 \mathrm{~m}$ wide (Figure 2c) and is large enough to support multiple users sitting around it (Figure 9).

\section{FABRICATING PNEUMATIC TRUSSES}

We employ a printer design for heat sealing fabrics that is similar to the designs from Aeromorph [20] and Printflatables [25]. To print custom truss members, we fit a narrow heated rod (implemented using a soldering iron) to the motion platform of a modified Cartesian 3D printer with an extended 4 feet by 1 foot fabrication area as illustrated by (Figure 10a). The printer applies heat at a constant rate to seal TPU-coated fabrics forming an inflated bladder. All examples use 200 Denier $^{4}$ Oxford heat-sealable cloth.

\subsection{Design Workflow}

We used The Rhinoceros CAD system (Rhino) with the Grasshopper visual programming plug-in to model our truss frames. All of our truss examples-dome, canopy, and table- were modeled using built-in sketching features such as line and poly-line to design a 3D model of a truss frame. Once an initial model was established, we verified the structural performance using Finite Element Analysis (FEA) with the Karamba3D plugin and performed

\footnotetext{
${ }^{4} \mathrm{~A}$ Denier is a unit of measure for textiles which refers to linear mass density of fibers.
} 


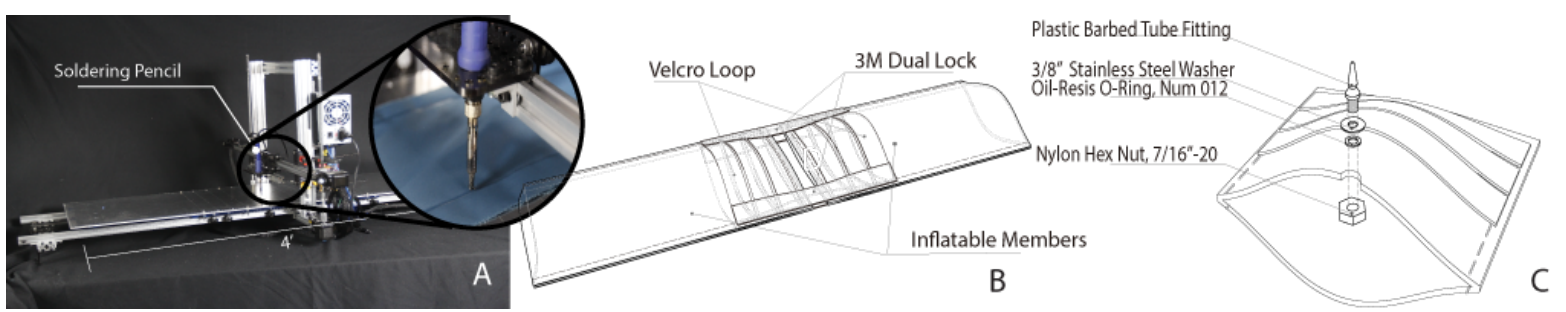

Fig. 10. (a) Our customized heat sealing printer; Working condition of the soldering iron can be seen through inset. (b) Joints made between inflatable members with velcro hook and loop. (c) Exploded view of the composition of the inlet valve system.

several iterations (manually) by changing triangle lengths/arrangement (along with FEA) to arrive at a final design. We then used Silkworm (Rhino plugin) to generate G-code paths for specific lengths of truss members. Once G-codes were generated, we fabricated the individual members using our custom printer. In the future, automatic support (with a design tool) can help in minimizing design iterations involved in finding the optimal configuration (length of truss members) while automatically checking for stability with FEA.

\subsection{Making Joints and Connections with Inflated Members}

We experimented with various mechanisms for forming joints between our truss members. The main criteria for forming our joints include making them lightweight so that they are able to move the members in the air. We investigated $\mathrm{CNC}$ machined foam blocks as a way of digitally fabricating the joints. However, we found that joint using hook and loop fasteners (Figure 10b) performed better as they are able to resist tension and still provide one-degree-of freedom revolute joints.

We used the $3 \mathrm{M}$ dual lock SJ3571 $1^{5}$ as a loop and the $3 \mathrm{M}$ (Type 250) $\mathrm{SJ} 3552 \mathrm{CF}^{6}$ as a hook component to form our connections (Figure 10b), providing a 12 in 2 contact area. Based on the data provided by the manufacturer, the hook and loop fasteners can resist $2.2 \mathrm{lb}$ of lateral force per square inch away from each other. Thus, with this contact area, we estimate each of our joints is capable of bearing a $26.4 \mathrm{lb}$ tension load.

\subsection{Installing Valves and Interconnected Tubes}

All our inflated members are made with inlet valves, hex nuts and washers (Figure 10c). Further, each of our truss members ( 5 inches wide) is installed with multiple valves and share a single pathway for air to allow inflation of the whole structure from a single point. However, with larger structures, such as the geodesic dome, we observed that providing multiple pathways for air and sequencing the airflow results in faster deployment. Our geodesic dome has six pathways of air while other examples (canopy and table) each have two pathways for air.

\subsection{Pneumatic Control System}

We used a Dewalt stationary air compressor that can reach up to $165 \mathrm{psi}$ and has a tank storage capacity of upto 6 gallons. We sensed the air pressure in the entire structure using a pressure sensor and regulated air passage $12 \mathrm{~V}$ DC solenoid valves. Further, we used a simple bang-bang control method for regulating the air pressure. Similarly, for actuation system, we used 12V DC solenoid valves controlled by an external power supply and an Arduino for digital logic. The pneumatic control system is connected to our input sensing/machine learning pipeline and responds to user action by controlling the solenoids.

\footnotetext{
${ }_{5}^{5}$ M dual lock SJ3571: https://multimedia.3m.com/mws/media/107979O/scotchmate-reclosable-fasteners-sj3571-and-sj3572.pdf

${ }^{6} 3 \mathrm{M}$ (Type 250) SJ3552CF: https://multimedia.3m.com/mws/media/349929O/dual-locktm-reclosable-fasteners-sj3551-sj3552-sj3550.pdf
} 


\section{EVALUATION}

We performed overall three types of evaluation: Sensing Evaluation, Design Workshop, and Strength Evaluation. As a part of our sensing evaluation, we chose to only evaluate ultrasonic acoustic sensing since it has never been implemented on inflatable objects and its robustness has never been studied on inflatable objects. Whereas other types of sensing techniques like capacitive and pressure sensing have been thoroughly studied in HCI literature [7] but missing engineering implementation with inflatable objects which we provide (section 4.3 and 4.4). Second, we follow up the sensing evaluation with a design workshop consisting of an informal gesture elicitation and sketching session with subjects who are expert designers. The overall goal of the design workshop is to uncover existing affordances with deployable structures and to generatively brainstorm novel uses that users are interested in exploring or building. Finally, we perform a preliminary strength analysis of our inflated truss structures to illustrate the approximate load bearing capacity of our structures. Combined together all these evaluations provide a design knowledge on choosing the type of applications when designing a new interactive deployable structure.

\subsection{Sensing Evaluation}

We evaluated the gesture recognition rates of our swept frequency ultrasonic acoustic sensing in the inflated trusses through a small user study consisting of 12 participants ( 8 male / 4 female; mean age: 27.33; age standard deviation: 3.23). All our participants were students at our university recruited through word-of-mouth.

We inflated a single truss member and attached two contact piezo elements at one end of the truss. The inflated member was affixed at each end to the table to simulate its placement in a structure and to maintain a stable interaction surface between users.

We trained a single model using ten samples for each gesture (swipe, knock, and squeeze), in a noisy and high traffic lab space. Additionally, we trained a null state (no interaction) during which there was no interaction with the inflated member, resulting in four possible classes for the classifier.

We evaluated the classifier on the 12 participants with no additional training from the participants. Each participant performed each gesture (knock, swipe, and squeeze) 10 times in a random order amounting to 30 trials per participant, and 360 trials in total. Prior to the study, participants were only told the name of the gestures and then asked to perform each gesture according to its name.

We found some participants swiped rapidly a single time, while others swiped slowly and multiple times (appearing to be more of a rub). Similarly, when knocking some participants knocked very hard once or twice while others knocked more gently for five to six times. However, these variations in gesturing across users had little effect on our model. As seen in Table 1, our classifier obtained an overall accuracy of $90.33 \%$. In one instance, a participant performed two swipe gestures that were not recognized (i.e. the classifier returned no interaction).

This was due to the user not applying enough pressure on the truss during the gesture to change the frequency response. The relatively high accuracy of our single generic model with minimal training suggests this sensing technique is well-suited to support new and different users interacting with these deployable structures without individualized training.

\subsection{Design Workshop}

We conducted an informal gesture elicitation [29] session in order to explore the interaction technique design space opened by our basic input sensing methods and to better understand whether this basic gesture vocabulary provides a good basis for building larger interactions. In the second part of the design workshop, we invited participants for sketching sessions with subjects who are expert designers. The goal was to judge whether 
Table 1. Confusion matrix (in percentages) for our cross-user swept frequency ultrasonic acoustic sensing user study. Our model was trained on a single user and tested with 12 participants for 360 trials in total. The classifier obtained an overall accuracy of $90.33 \%$.

\begin{tabular}{|c|c|c|c|}
\hline \multicolumn{3}{|c|}{ Performed Gesture } & \multirow[b]{2}{*}{ Classified Gesture } \\
\hline swipe & knock & squeeze & \\
\hline 84 & 1 & 3 & swipe \\
\hline 8 & 93 & 3 & knock \\
\hline 6 & 6 & 94 & squeeze \\
\hline 2 & 0 & 0 & no interaction \\
\hline
\end{tabular}

participants could create diverse scenarios in which interactive deployable structures could be used along with their interaction. During the second part, the participants were free to sketch.

8.2.1 Participants: We recruited 5 participants from our university (2 male, 3 female, aged 27-35). Two majored in design, 1 in architecture and the other two had professional industrial design backgrounds. All participants reported to have been using mobile phones/tablets with gesture uses every day except one participant said he uses gestures uses 2-3 times a week. All participants also reported having sketching experience.

8.2.2 Tasks \& Procedure: The workshop took place in our research lab and lasted between 30 and 45 minutes for each participant. Participants were given empty sheets or paper, pencil and single inflated member. To begin, participants were given an introduction into the project by the experimenter on some basic understanding of what deployable structures are and were shown various parts of the structures that can actuate. The participants were instructed to only perform gestures or interactions on the inflated member given and were asked to think aloud when performing gestures. Participants were shown 3 pictures of interactive tasks from the examples ((Figure 2a) and (Figure 2b)) available in the paper namely - 1) geodesic dome door opens upon input (Figure 4 a \& b) 2) window of the dome rolls after input (Figure $4 \mathrm{c} \& \mathrm{~d}$ ) and 3) controlling the blinds of the canopy (Figure 8) using input. Note that in all three of these pictures of interaction, we masked the user performing input using a gesture. We only showed pictures of the structure and their desired responses such as door opened, window opened or shades rotated, etc. For each interaction, we asked users to perform 2-3 gestures on the inflated member and point to the part of the structure would they perform the gesture on. After finishing the gesture elicitation, we showed participants the video of our system with various inputs, outputs and interactions along with three examples (Figure 2) we constructed, we then asked them to generatively come up with 2-3 new designs of interactive deployable structures by sketching them.

8.2.3 Gestures Analysis: Participants proposed a total of 40 interactions for the 3 referents - opening the door, opening the window and controlling blinds, 30 of which were distinct (considering distinctness on a per-referent basis). Specifically for opening the door, in total 15 gestures (11 distinct) were proposed, for opening a window - 11 (9 distinct) were proposed and controlling blinds - 14 (10 distinct) were proposed. Most popular among overlapping gestures were - for opening door was tap multiple times (2/total) and squeeze (2/total), popular for controlling blinds is slide/swipe (3/total) and for opening windows of the dome there was no clear majority consensus.

We observed three main themes in gestures: participants sometimes used - multiple hands to engage in interactions, 


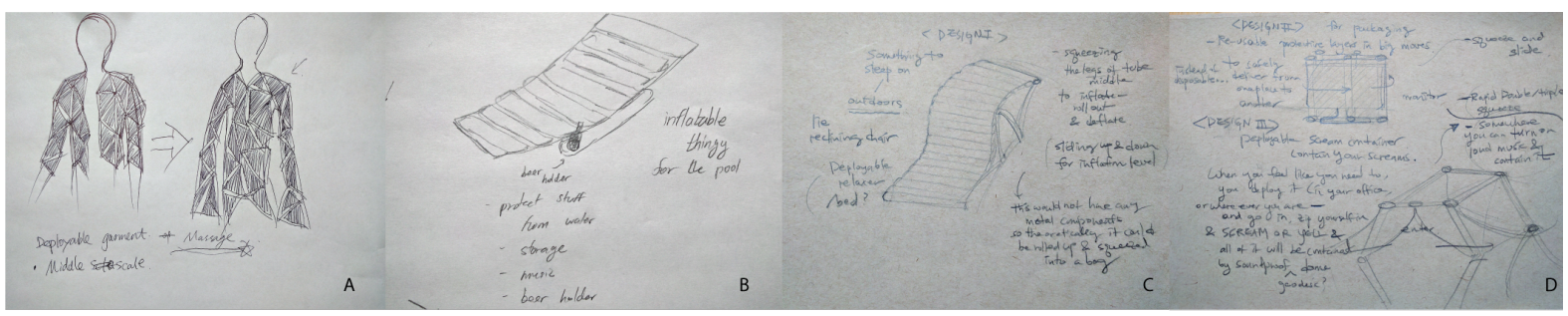

Fig. 11. Shows various user sketches of interactive deployable structures a) Shows a deployable vest that can custom fit upon input b) shows a floating pool chair with adjustable storage closet c) shows a chair with adjustable backrest d) shows various other interactive deployable structures

performed input on multiple inflated members sequentially and combined two gestures at once - e.g., press and slide. Most of these interactions can be already supported by one or more input methods proposed in the paper. For instance squeeze and slide can be implemented by combining pressure and capacitive sensing together. Similarly interacting with multiple inflated members at once can be sensed using pressure sensing.

Participants observed that different parts of the structure afforded different interaction. For instance P2 mentioned "I'm more comfortable performing these gestures (showing the gesture) inside than outside since people are able to see me", similarly, P4 mentioned that "squeeze is better afforded in this part (pointing) to the structure, since there is a hanging inflated member rather than inflated member attached to the structure". These reactions suggest that participants are selective about which parts of the structure they want to interact with.

8.2.4 Sketches of Interactive Deployable Structures: After watching the video of interactive deployable structures (attached supplemental video), showing various input, output and construction possibilities, we asked participants to brainstorm and sketch 2-3 designs they were interested in building. The majority of the participants proposed at least 1 water based interactive deployable structure that they could build. For instance, $2 / 5$ participants suggested inflatable pool chair that has a storage closet that can be deployed for drinks (Figure 11b). Other water-based inflatable interactive structures were a boat that had interactive control for rudder, swimming safety ring, an inflatable interactive hammock, interactive deployable fishing net that transforms to catch a fish, chair that has an adjustable backrest (Figure 11c), deployable garment (Figure 11a) that can custom fit itself, etc. Below are some of the sketches of what participants wanted to build.

\subsection{Strength Analysis}

In order to assess the structural strength of our prototypes, we performed finite element analysis (FEA). To perform FEA, we first gathered the material properties such as elastic modulus (a measure of the intrinsic stiffness of material) by performing compression analysis using a universal testing machine (Instron 5969) on truss elements (Figure 12a).

We compressed our truss members at different pressures: 16, 17, 18, and 19 psi (max). Our experiments (Figure 12b), reveal that at 19 psi pressure the truss element can carry up to $142 \mathrm{~N}$ load before buckling while this maximum load drops to $91 \mathrm{~N}$ at 16 psi pressure. Hence, we recommend that the truss members be maintained at 19 psi for maximum load bearing strength and utilized the elastic modulus at 19 psi for our FEA modeling.

To further proceed, we used Karamba3D software package along with Grasshopper to model and perform our FEA analysis. During the modeling, we assumed all our members are connected with revolute joints, which allow members to rotate around the joint but otherwise constraints relative movement. Based on these assumptions our truss structure allows truss members to bear only axial loads, rather than bending or torsion. 

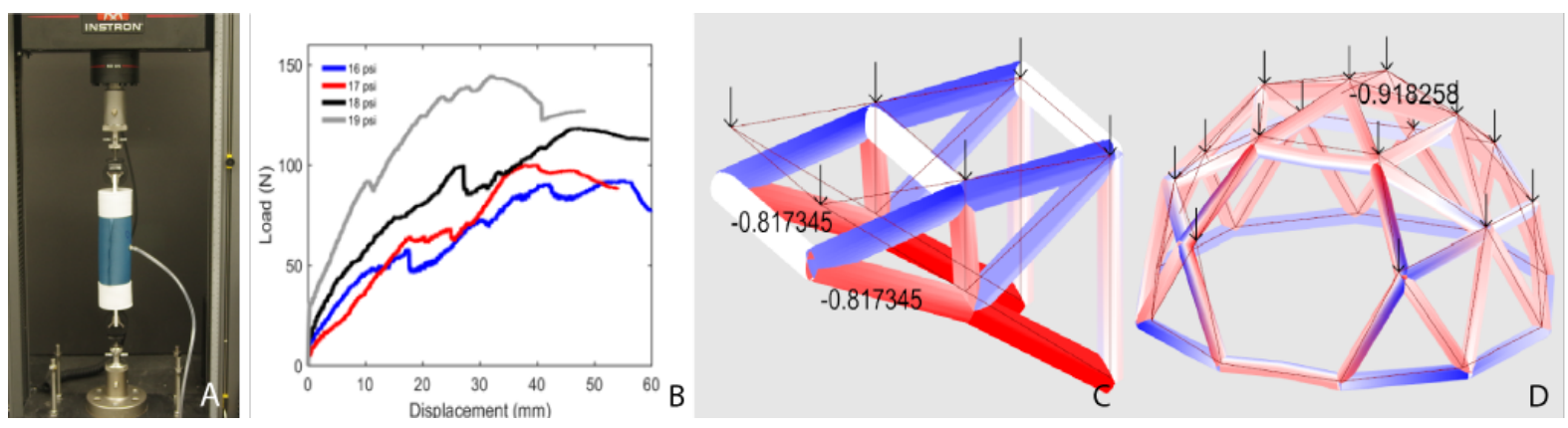

Fig. 12. (a) Compression testing of an inflated truss member with an Instron 5969 testing machine. (b) Shows loading vs applied pressure behavior of truss elements under compressive load. (c) FEA analysis depicting the deflection of our truss frames from undeformed state. (d) Dome shows a deflection of $0.92 \mathrm{~cm}$ from top and canopy shows a deflection of $0.81 \mathrm{~cm}$ from tip of the roof.

Finally, to find the maximum load of the entire structure can bear before reaching the maximum deflection, we first referenced the International Building $\mathrm{Code}^{7}$ for maximum deflection criteria. The code gives specific evaluation schemes for our structures: $\mathrm{L} / 360$ (floor structure) and L/180 (roof structure), where $\mathrm{L}$ is the diameter of the dome and in the case of canopy it is the length of the roof.

Applying these criteria over the dimensions (the diameter of the dome is $3.3 \mathrm{~m}$ and the length of the canopy is $2.9 \mathrm{~m}$ ) gives us maximum allowable deflection of $0.92 \mathrm{~cm}$ for the dome and $0.81 \mathrm{~cm}$ for the canopy (Figure $12 \mathrm{c}$ ). We used these two criteria to find the maximum load for both our structures.

During our analysis, we applied simulated point loads to the joints of the canopy and the dome. In the results of FEA analysis, as seen in (Figure $12 \mathrm{~d}$ ), the members in tension are rendered in black and compression are rendered in red. Further, the deformation of the shape is compared with the original shape (represented as solid lines).

Maximum deflection is displayed in centimeters. This analysis predicts that the dome can bear $7.6 \mathrm{~kg}(507 \mathrm{~g}$ at each joint) and the canopy can bear $1.88 \mathrm{~kg}$ (313g at each top joint). While this analysis is in no sense complete, it indicates that acceptably strong structures can be fabricated with these techniques.

\section{LIMITATIONS AND FUTURE WORK}

Our work shows a range of techniques that enable the custom fabrication of room-scale deployable pneumatic structures with integrated input and actuation capabilities. This approach opens up many new opportunities. However, it also has limitations. For example, while we are able to assemble trusses of arbitrary sizes from shorter trusses (limited to by the length of our printer), the effort involved in assembly depends in part on the printer size. One way to address this limitation is to further automate the joinery of inflatable truss members. Also, in all our examples and prototypes we used a regulated pressure set-up with constant air supply to keep the structures intact. One way to eliminate the need for pressure regulation is to use a rigidization strategy such as injecting foam throughout connected members to form the final shapes. We are also interested in examining how wrapping our inflatable members with fiber-reinforced composites and curing them on-demand can be used to rigidify the structure.

\footnotetext{
${ }^{7}$ International Building Code 2017: https://codes.iccsafe.org/public/document/toc/542/
} 


\section{CONCLUSION}

In this paper, we explored the design space of deployable pneumatic structures with integrated interactive capabilities that can be digitally fabricated at room-scale. We specifically looked at embedding three types of sensing in our objects - acoustic, capacitive and pressure, to provide diverse forms of input. Our example prototypes such as a geodesic dome, canopy, and portable table demonstrated how users can leverage these interaction possibilities as part of an environment which is responsive to human input. Further, our structural analysis (FEA) indicates that our structures can handle overall loads of $7.6 \mathrm{~kg}$ for the dome and $1.8 \mathrm{~kg}$ for the canopy. Finally, our lab-scale study on the inflated truss members revealed that we are able to classify gestures such as knock, squeeze, swipe and no interaction with an overall accuracy of $90 \%$. Furthermore, an exploratory design workshop conducted with users provided a pathway to understand whether the basic gesture vocabulary proposed forms good bases for larger interactions and design space. From sensing to actuation, we have demonstrated an approach to fabricating room-scale, deployable pneumatic structures that possess interactivity which is fabricated as part of the object. Our work builds on prior efforts related to deployable and relocatable objects and allows for human interaction to take place on these structures in a big way.

\section{ACKNOWLEDGMENTS}

We would like to acknowledge Siyan Zhao for her advise in running the user studies. We would like thank all our user study participants. This work was funded in part by the National Science Foundation under grant IIS-1718651.

\section{REFERENCES}

[1] 2018. Mataerial. http://mataerial.com. [Online; accessed 2018-04-05].

[2] 2018. Minibuilders. http://iaac.net/printingrobots. [Online; accessed 2018-04-05].

[3] Moritz Bächer, Benjamin Hepp, Fabrizio Pece, Paul G Kry, Bernd Bickel, Bernhard Thomaszewski, and Otmar Hilliges. 2016. Defsense: Computational design of customized deformable input devices. In Proceedings of the $2016 \mathrm{CHI}$ Conference on Human Factors in Computing Systems. ACM, 3806-3816.

[4] Alan Bränzel, Christian Holz, Daniel Hoffmann, Dominik Schmidt, Marius Knaust, Patrick Lühne, René Meusel, Stephan Richter, and Patrick Baudisch. 2013. GravitySpace: Tracking Users and Their Poses in a Smart Room Using a Pressure-sensing Floor. In Proceedings of the SIGCHI Conference on Human Factors in Computing Systems (CHI '13). ACM, New York, NY, USA, 725-734. https://doi.org/10.1145/2470654.2470757

[5] Charis J Gantes. 2001. Deployable structures: analysis and design. Wit Press.

[6] Fabio Gramazio, Matthias Kohler, and Silvan Oesterle. 2010. Encoding material. Architectural Design 80, 4 (2010), 108-115.

[7] Tobias Grosse-Puppendahl, Christian Holz, Gabe Cohn, Raphael Wimmer, Oskar Bechtold, Steve Hodges, Matthew S Reynolds, and Joshua R Smith. 2017. Finding common ground: A survey of capacitive sensing in human-computer interaction. In Proceedings of the 2017 CHI Conference on Human Factors in Computing Systems. ACM, 3293-3315.

[8] Beverly L Harrison, Kenneth P Fishkin, Anuj Gujar, Carlos Mochon, and Roy Want. 1998. Squeeze me, hold me, tilt me! An exploration of manipulative user interfaces. In Proceedings of the SIGCHI conference on Human factors in computing systems. ACM Press/AddisonWesley Publishing Co., 17-24.

[9] Alexandra Ion, Johannes Frohnhofen, Ludwig Wall, Robert Kovacs, Mirela Alistar, Jack Lindsay, Pedro Lopes, Hsiang-Ting Chen, and Patrick Baudisch. 2016. Metamaterial mechanisms. In Proceedings of the 29th Annual Symposium on User Interface Software and Technology. ACM, 529-539.

[10] Hiroshi Ishii, Craig Wisneski, Julian Orbanes, Ben Chun, and Joe Paradiso. 1999. PingPongPlus: design of an athletic-tangible interface for computer-supported cooperative play. In Proceedings of the SIGCHI conference on Human Factors in Computing Systems. ACM, $394-401$.

[11] Robert Kovacs, Anna Seufert, Ludwig Wall, Hsiang-Ting Chen, Florian Meinel, Willi Müller, Sijing You, Maximilian Brehm, Jonathan Striebel, Yannis Kommana, et al. 2017. Trussfab: Fabricating sturdy large-scale structures on desktop 3d printers. In Proceedings of the 2017 CHI Conference on Human Factors in Computing Systems. ACM, 2606-2616.

[12] Benjamin Lafreniere, Tovi Grossman, Fraser Anderson, Justin Matejka, Heather Kerrick, Danil Nagy, Lauren Vasey, Evan Atherton, Nicholas Beirne, Marcelo H Coelho, et al. 2016. Crowdsourced fabrication. In Proceedings of the 29th Annual Symposium on User Interface Software and Technology. ACM, 15-28. 
[13] Gierad Laput, Eric Brockmeyer, Scott E Hudson, and Chris Harrison. 2015. Acoustruments: Passive, acoustically-driven, interactive controls for handheld devices. In Proceedings of the 33rd Annual ACM Conference on Human Factors in Computing Systems. ACM, $2161-2170$

[14] Joanne Leong, Patrick Parzer, Florian Perteneder, Teo Babic, Christian Rendl, Anita Vogl, Hubert Egger, Alex Olwal, and Michael Haller. 2016. proCover: sensory augmentation of prosthetic limbs using smart textile covers. In Proceedings of the 29th Annual Symposium on User Interface Software and Technology. ACM, 335-346.

[15] Linjie Luo, Ilya Baran, Szymon Rusinkiewicz, and Wojciech Matusik. 2012. Chopper: partitioning models into 3D-printable parts. (2012).

[16] Jörg Müller, Dieter Eberle, and Constantin Schmidt. 2015. Baselase: An interactive focus + context laser floor. In Proceedings of the 33rd Annual ACM Conference on Human Factors in Computing Systems. ACM, 3869-3878.

[17] Satoshi Nakamaru, Ryosuke Nakayama, Ryuma Niiyama, and Yasuaki Kakehi. 2017. FoamSense: Design of Three Dimensional Soft Sensors with Porous Materials. In Proceedings of the 30th Annual ACM Symposium on User Interface Software and Technology. ACM, 437-447.

[18] Ryuma Niiyama, Daniela Rus, and Sangbae Kim. 2014. Pouch motors: Printable/inflatable soft actuators for robotics. In Robotics and Automation (ICRA), 2014 IEEE International Conference on. IEEE, 6332-6337.

[19] Simon Olberding, Michael Wessely, and Jürgen Steimle. 2014. PrintScreen: fabricating highly customizable thin-film touch-displays. In Proceedings of the 27th annual ACM symposium on User interface software and technology. ACM, 281-290.

[20] Jifei Ou, Mélina Skouras, Nikolaos Vlavianos, Felix Heibeck, Chin-Yi Cheng, Jannik Peters, and Hiroshi Ishii. 2016. aeroMorph-heatsealing inflatable shape-change materials for interaction design. In Proceedings of the 29th Annual Symposium on User Interface Software and Technology. ACM, 121-132.

[21] Joseph A Paradiso, Kai-yuh Hsiao, Joshua Strickon, Joshua Lifton, and Ari Adler. 2000. Sensor systems for interactive surfaces. IBM Systems Journal 39, 3 (2000), 892-914.

[22] Joseph A Paradiso, Che King Leo, Nisha Checka, and Kaijen Hsiao. 2002. Passive acoustic sensing for tracking knocks atop large interactive displays. In Sensors, 2002. Proceedings of IEEE, Vol. 1. IEEE, 521-527.

[23] Ivan Poupyrev, Nan-Wei Gong, Shiho Fukuhara, Mustafa Emre Karagozler, Carsten Schwesig, and Karen E Robinson. 2016. Project Jacquard: interactive digital textiles at scale. In Proceedings of the $2016 \mathrm{CHI}$ Conference on Human Factors in Computing Systems. ACM, 4216-4227.

[24] Graham Robinson and J Bruce C Davies. 1999. Continuum robots-a state of the art. In Robotics and Automation, 1999. Proceedings. 1999 IEEE International Conference on, Vol. 4. IEEE, 2849-2854.

[25] Harpreet Sareen, Udayan Umapathi, Patrick Shin, Yasuaki Kakehi, Jifei Ou, Hiroshi Ishii, and Pattie Maes. 2017. Printflatables: printing human-scale, functional and dynamic inflatable objects. In Proceedings of the 2017 CHI Conference on Human Factors in Computing Systems. ACM, 3669-3680.

[26] Robert F Shepherd, Filip Ilievski, Wonjae Choi, Stephen A Morin, Adam A Stokes, Aaron D Mazzeo, Xin Chen, Michael Wang, and George M Whitesides. 2011. Multigait soft robot. Proceedings of the national academy of sciences 108, 51 (2011), 20400-20403.

[27] Anita Vogl, Patrick Parzer, Teo Babic, Joanne Leong, Alex Olwal, and Michael Haller. 2017. StretchEBand: Enabling Fabric-based Interactions through Rapid Fabrication of Textile Stretch Sensors.. In CHI. 2617-2627.

[28] Karl Willis, Eric Brockmeyer, Scott Hudson, and Ivan Poupyrev. 2012. Printed optics: 3D printing of embedded optical elements for interactive devices. In Proceedings of the 25th annual ACM symposium on User interface software and technology. ACM, 589-598.

[29] Jacob O Wobbrock, Meredith Ringel Morris, and Andrew D Wilson. 2009. User-defined gestures for surface computing. In Proceedings of the SIGCHI Conference on Human Factors in Computing Systems. ACM, 1083-1092.

[30] Lining Yao, Ryuma Niiyama, Jifei Ou, Sean Follmer, Clark Della Silva, and Hiroshi Ishii. 2013. PneUI: pneumatically actuated soft composite materials for shape changing interfaces. In Proceedings of the 26th annual ACM symposium on User interface software and technology. ACM, 13-22.

[31] Sang Ho Yoon, Ke Huo, Yunbo Zhang, Guiming Chen, Luis Paredes, Subramanian Chidambaram, and Karthik Ramani. 2017. iSoft: A Customizable Soft Sensor with Real-time Continuous Contact and Stretching Sensing. In Proceedings of the 30th Annual ACM Symposium on User Interface Software and Technology. ACM, 665-678.

[32] Hironori Yoshida, Takeo Igarashi, Yusuke Obuchi, Yosuke Takami, Jun Sato, Mika Araki, Masaaki Miki, Kosuke Nagata, Kazuhide Sakai, and Syunsuke Igarashi. 2015. Architecture-scale human-assisted additive manufacturing. ACM Transactions on Graphics (TOG) 34, 4 (2015), 88.

[33] Yang Zhang, Chouchang Jack Yang, Scott E Hudson, Chris Harrison, and Alanson Sample. 2018. Wall++: Room-Scale Interactive and Context-Aware Sensing. In Proceedings of the 2018 CHI Conference on Human Factors in Computing Systems. ACM, 273.

Received November 2018; revised February 2019; accepted April 2019 